\title{
ESTRATEGIA PARA MEJORAR LA CALIDAD DE VIDA DE LOS HABITANTES, CASO: COMUNIDAD CUNUYACU, PROVINCIA DE TUNGURAHUA (ECUADOR)
}

\section{STRATEGY TO IMPROVE THE QUALITY OF LIFE OF INHABITANTS, CASE: COMMUNITY CUNUYACU, PROVINCE OF TUNGURAHUA (ECUADOR)}

\author{
Franqui Fernando Esparza Paz*, Cristina Nataly Villegas Freire**, \\ Cristian German Santiana Espín***, Carmita Isabel Ajitimbay Muñoz ${ }^{* * *}$
}

\footnotetext{
* Ingeniero de Empresas. Máster en Dirección de Empresas, mención Proyectos. Docente investigador Facultad de Administración de Empresas. Escuela Superior Politécnica de Chimborazo (Ecuador).

** Magíster en Formulación, Evaluación y Gerencia de Proyectos para el Desarrollo. Docente investigadora Escuela de Industrias Pecuarias e Ingeniería Zootécnica, Facultad de Ciencias Pecuarias. Escuela Superior Politécnica de Chimborazo (Ecuador).

*** Magíster en Formulación, Evaluación y Gerencia de Proyectos para el Desarrollo. Docente investigador en la Escuela de Ingeniería Mecánica, Facultad de Mecánica. Escuela Superior Politécnica de Chimborazo (Ecuador).

**** Economista con mención en Gestión Empresarial. Magíster en Gestión y Desarrollo Social. Docente investigadora Facultad de Administración de Empresas. Escuela Superior Politécnica de Chimborazo (Ecuador).
}

Dirección para recibir correspondencia: ffespaz@yahoo.es 
ESTRATEGIA PARA MEJORAR LA CALIDAD DE VIDA DE LOS HABITANTES, CASO: COMUNIDAD

CUNUYACU, PROVINCIA DE TUNGURAHUA (ECUADOR)

\section{RESUMEN}

OBJETIVO: La investigación tuvo como objetivo demostrar como a través de los proyectos de desarrollo turístico sostenibles se puede mejorar la calidad de vida de los habitantes.

MATERIALES Y MÉTODOS: El estudio se realizó en el Complejo de Aguas Termales de la comunidad Cunuyacu, Cantón Ambato, provincia de Tungurahua (Ecuador), que cuenta con potenciales fuentes naturales para recibir a los visitantes. Para la recopilación de la información se utilizó como instrumento la encuesta, se tomó como muestra a turistas nacionales y extranjeros que visitan la Reserva de Producción de Flora y Fauna Chimborazo (RPFFCH). Se aplicó los métodos inductivo-deductivo, histórico y dialéctico, y técnicas de investigación de campo y documental-bibliográfica, a nivel exploratorio, descriptivo, analítico y prospectivo.

RESULTADOS: Se determinó la demanda insatisfecha del servicio turístico y se analizó los indicadores económicos captando una demanda objetiva en tres escenarios que permitieron observar que el proyecto es viable con una Tasa Interna de Retorno del $21.16 \%$ y un Valor Actual Neto de $\$ 108.673,56$. La implementación del proyecto no afecta al ambiente, ni a la cultura y tradiciones de la comunidad.

CONCLUSIÓN: Los proyectos de desarrollo turístico permiten mejorar la calidad de vida de los habitantes, entre otros, en temas de economía, salud, educación. Con la evaluación sociocultural y ambiental, se puede concluir que el proyecto producirá un impacto positivo a nivel cultural, social y ambiental.

PALABRAS CLAVE: Proyecto. Turismo. Comunitario. Estrategia. Desarrollo. Calidad. 
ESTRATEGIA PARA MEJORAR LA CALIDAD DE VIDA DE LOS HABITANTES, CASO: COMUNIDAD CUNUYACU, PROVINCIA DE TUNGURAHUA (ECUADOR)

\section{ABSTRACT}

OBJECTIVE: The objective of the research was to demonstrate how through sustainable tourism development projects the quality of life of the inhabitants can be improved.

MATERIALS AND METHODS: The study was conducted in the Thermal Waters Complex of the Cunuyacu community, Ambato Canton, Tungurahua province (Ecuador), which has potential natural sources to receive visitors. To collect the information, the survey was used as an instrument, and national and foreign tourists visiting the Chimborazo Flora and Fauna Production Reserve (RPFFCH) were sampled. The inductive-deductive, historical and dialectical methods, and field and documentary-bibliographic research techniques were applied at the exploratory, descriptive, analytical and prospective levels.

RESULTS: The unsatisfied demand of the tourist service was determined and the economic indicators were analyzed capturing an objective demand in three scenarios that allowed observing that the project is viable with an Internal Rate of Return of $21.16 \%$ and a Net Present Value of $\$ 108,673.56$. The implementation of the project does not affect the environment, nor the culture and traditions of the community.

CONCLUSION: The tourism development projects allow to improve the quality of life of the inhabitants, among others, in subjects of economy, health, education. With the socio-cultural and environmental assessment, it can be concluded that the project will produce a positive impact at a cultural, social and environmental level.

KEYWORDS: Project. Tourism. Community. Strategy. Development. Quality.

\section{INTRODUCCIÓN}

El interés por la calidad de vida ha existido desde tiempos inmemoriales. La aparición del concepto como tal y la preocupación por la evaluación sistemática y científica del mismo es relativamente reciente. La idea comienza a generalizarse en la década de los 60 hasta convertirse hoy en un concepto utilizado en diferentes ámbitos, como son: la salud, la salud mental, la educación, la economía, la política y el mundo de los servicios en general. (Revista Mexicana de Medicina Física y Rehabilitación 2006). 
ESTRATEGIA PARA MEJORAR LA CALIDAD DE VIDA DE LOS HABITANTES, CASO: COMUNIDAD CUNUYACU, PROVINCIA DE TUNGURAHUA (ECUADOR)

Para Casas (1999) la extrapolación de la calidad humana individual a la social catapultó rápidamente el concepto calidad de vida como valor, pero también como idea aglutinadora de aspiraciones colectivas ampliamente compartidas, de anhelos de cambio social a mejor. A partir de ahí, el reto social de la calidad, se implanta como un objetivo, tanto para el desarrollo de políticas, como de intervenciones profesionales en muchos ámbitos, incluido particularmente el de las políticas sociales en su más amplia acepción.

El problema central del presente estudio se enfoca en ¿Cómo influye la puesta en marcha de un proyecto de desarrollo turístico sostenible para un Complejo de Aguas Termales en la comunidad de Cunuyacu, en la calidad de vida de sus habitantes? La estrategia de investigación es medir cómo la implementación y aplicación del proyecto ayuda a mejorar las condiciones de vida de los pobladores del sector. El objetivo del estudio, consiste en desarrollar un proyecto de desarrollo turístico sostenible en el Complejo de Aguas Termales de la comunidad Cunuyacu, que permita mejorar la calidad de vida de los habitantes de la zona, mediante un trabajo de campo con los principales actores de la localidad.

La comunidad de Cunuyacu, perteneciente a Cantón Ambato, en la provincia de Tungurahua (Ecuador), es un sector con gran potencial para emprender en el desarrollo local, con altas expectativas para ofertar turismo comunitario con valor agregado como son las fuentes de aguas termales para el desarrollo de turismo de salud destinado a brindar propiedades curativas a los visitantes, posee gran variedad de atractivos, paisajes, producción agrícola y animal, cultura ancestral y en general por todo lo que representa la parroquia, en el futuro llegar a ser uno de los lugares más visitados por turistas nacionales y extranjeros.

El turismo sostenible plantea oportunidades para mejorar las condiciones de vida de los seres humanos, ya sea a través de la articulación de negocios privados y comunitarios en cadenas de valor que generan empleo directo e indirectamente, como a través de la demanda de servicios turísticos que generen experiencias auténticas. Los desafíos planteados para luchar contra la pobreza a través del turismo incluyen: 1. Promover el turismo como instrumento de reducción de la pobreza a través de la sensibilización de todos los actores; 2. Encontrar mejores maneras de canalizar el gasto de los visitantes hacia los pobres y economías locales; 3. Demostrar la eficacia del turismo en la lucha contra la pobreza con proyectos piloto y su escalamiento; 4. Promover esos proyectos y multiplicarlos (Organización Mundial del Turismo, 2007). 
ESTRATEGIA PARA MEJORAR LA CALIDAD DE VIDA DE LOS HABITANTES, CASO: COMUNIDAD CUNUYACU, PROVINCIA DE TUNGURAHUA (ECUADOR)

La inestabilidad económica que viene soportando el Ecuador por la baja del precio del petróleo como principal fuente de ingresos de la economía ecuatoriana, ha afectado a todos los sectores económicos y particularmente al turístico; lo cual en los últimos años, ha centrado su atención en la industria del turismo por su aporte en la creación de empleo directo e indirecto, la generación de divisas, redistribución de la riqueza, permitiendo albergar a turistas nacionales y extranjeros que deseen disfrutar momentos únicos de diversión, descanso y esparcimiento.

Para analizar el aporte del sector, de acuerdo a la Coordinación General de Estadísticas del Ministerio de Turismo, en el Ecuador el turismo interno durante el año 2016 ha generado 12.3 millones de viajes, el $40 \%$ de estos viajes se realizaron durante los diferentes feriados lo que representó alrededor de 4.9 millones de viajes. Durante el feriado de fin del año 2016 e inicios del 2017 el país generó 618.972 viajes, generando un movimiento económico de alrededor de los 37,6 millones de dólares. Según la Revista Líderes (2016) las "visitas de turistas a Ecuador subieron $6,4 \%$ en el primer trimestre de 2015. Un total de 416.030 turistas arribó a Ecuador durante el primer trimestre de 2015, lo que evidencia un crecimiento del 6,4\% con relación a igual periodo de 2014 cuando llegaron 391.167 visitantes".

Para la Organización Mundial del Turismo (2007), el turismo es un fenómeno social, cultural y económico relacionado con el movimiento de las personas a lugares que se encuentran fuera de su lugar de residencia habitual por motivos personales o de negocios/profesionales. Estas personas se denominan visitantes (que pueden ser turistas o excursionistas; residentes o no residentes) y el turismo tiene que ver con sus actividades, de las cuales algunas implican un gasto turístico.

Baca (2010) indica que un proyecto es la búsqueda de una solución inteligente al planteamiento de un problema, la cual tiende a resolver una necesidad humana. En este sentido, puede haber diferentes ideas, inversiones de monto distinto, tecnología y metodologías con diverso enfoque, pero todas ellas destinadas a resolver las necesidades del ser humano en todas sus facetas, como pueden ser: educación, alimentación, salud, ambiente, cultura.

Varela (2010), manifiesta que las decisiones gerenciales tal vez más difíciles y de mayor incidencia son las decisiones de inversión en proyectos. Estas decisiones implican básicamente decidir sobre el tipo (que se va hacer), la forma (como se va hacer), el momento (cuando se va 
ESTRATEGIA PARA MEJORAR LA CALIDAD DE VIDA DE LOS HABITANTES, CASO: COMUNIDAD CUNUYACU, PROVINCIA DE TUNGURAHUA (ECUADOR)

hacer), con quien (quienes van a participar) y el monto (cuanto se va a invertir) de los recursos que se van a asignar a cada una de las actividades de desarrollo.

Actualmente, el Ecuador cuenta con un proyecto que impulsa el desarrollo socioeconómico del país, dentro de este el desarrollo turístico ha permitido la inclusión de sectores que no han sido antes atendidos, de manera que pueden participar directa o indirectamente obteniendo beneficios socio-económicos para su población, contribuyendo así al desarrollo y sostenibilidad de dichos sectores del país (Plan Nacional del Buen Vivir, 2013).

En este contexto, existe la expectativa que el turismo sostenible sea un instrumento efectivo para la creación de riqueza, la reducción de la pobreza y para la construcción de sociedades más democráticas y equitativas, sobre una base de respeto de los umbrales ecológicos y las culturas (Plandetur, 2007).

\section{MATERIALES Y MÉTODOS}

El presente trabajo, se llevó a cabo en la comunidad Cunuyacu, Parroquia Pilahuín, Cantón Ambato, provincia de Tungurahua; en la vía Ambato-Guaranda a 50km al Sur-Oeste de la capital de la provincia, donde el estudio se realizó utilizando los métodos de investigación: Dialéctico, mediante técnicas de investigación bibliográfica y de campo, a un nivel exploratorio, descriptivo, analítico y prospectivo.

El objetivo de desarrollar un proyecto turístico sostenible en el Complejo de Aguas Termales de la comunidad Cunuyacu, que permita mejorar la calidad de vida de los habitantes de la zona, se llevó a cabo en principio con la recopilación de información secundaria a través de mapas topográficos, estudios económicos de las entidades locales, regionales y nacionales de planificación y administración, información sobre estadísticas y censos, fotografías del territorio proporcionado por las dependencias locales y regionales y de sitios web.

Asimismo, se recabó información de la división política administrativa (provincia, cantón, parroquia, comunidad), límites de cantón, vías de acceso.

Por otro lado, se obtuvieron datos acerca de la etnicidad (nacionalidad y pueblo), historia, población (total de habitantes, número de familias, distribución por género y edad), datos sobre migración, nivel de instrucción, profesión u ocupación, servicios básicos disponibles (agua, energía, alcantarillado, recolección y tratamiento de desechos), vivienda (tipo, propiedad, ESPARZA-PAZ F.F., VILLEGAS FREIRE C.N., SANTIANA-ESPIN C.G., AJITIMBAY-MUÑOZ C.I. 
ESTRATEGIA PARA MEJORAR LA CALIDAD DE VIDA DE LOS HABITANTES, CASO: COMUNIDAD CUNUYACU, PROVINCIA DE TUNGURAHUA (ECUADOR)

características), salud, educación (tipo de establecimiento, niveles, número de estudiantes y docentes), medios de transporte (unidades, frecuencia, precio), comunicación, servicios sanitarios (tipo de baños, letrina, pozo ciego, pozo séptico), combustibles utilizados y el abastecimiento de productos.

En el ámbito ecológico territorial, se procedió a determinar las condiciones ambientales de la zona (clima, paisaje natural), usos de suelo, descripción general de flora y fauna especies en peligro de extinción, principales problemas ambientales.

En el ámbito económico productivo, se analizaron cuáles son las actividades económicas (tipo, ocupación) y el desarrollo actual del turismo en la zona.

En el ámbito político administrativo, se estableció cuál es la administración interna (organigrama, composición administrativa, funciones) y se determinó las instituciones que trabajan en la zona.

Para determinar si la formulación del proyecto de desarrollo turístico sostenible del Complejo de Aguas Termales de la comunidad Cunuyacu mejora la calidad de vida de los habitantes, se trabajó con el estimador estadístico Chi-cuadrado.

Para determinar la viabilidad comercial, se realizó el análisis de la demanda y la oferta. Para establecer la demanda se aplicaron encuestas, el objetivo de la misma fue investigar la disposición de visitas al complejo, el perfil y las preferencias de actividades turísticas de los visitantes. Para fines de la oferta se realizó un inventario de atractivos turísticos recopilando información de fuentes primarias (técnica de la observación de campo, para establecer el diagnóstico de la situación actual) y secundarias (información bibliográfica y documental existente en los Planes de Desarrollo del Gobierno Autónomo Descentralizado Parroquial).

Según Vara (2010) el universo o población "es el conjunto de todos los individuos (objetos, personas, documentos, data, eventos, empresas, situaciones, etc.) a investigar". El universo de estudio comprende el total de turistas nacionales y extranjeros que durante el año 2013 visitaron la reserva.

El cálculo de la muestra fue basado en Vara (2010), consecuentemente se efectuaron 382 encuestas, $85 \%$ fue dirigido a turistas nacionales, mientras que el $15 \%$ fue dirigido a turistas extranjeros. 
ESTRATEGIA PARA MEJORAR LA CALIDAD DE VIDA DE LOS HABITANTES, CASO: COMUNIDAD CUNUYACU, PROVINCIA DE TUNGURAHUA (ECUADOR)

Una vez recopilada la información, se procedió a la tabulación y procesamiento de datos, para el efecto se utilizó varias herramientas informáticas como el programa estadístico Statistical Package for the Social Sciences (SPSS) y excel, los mismos que permitieron sistematizar los datos e ilustrar en figuras y tablas.

El desarrollo del estudio exigió la utilización de los siguientes elementos: GPS, cámara fotográfica digital, memoria flash, computadora, impresora, registros de partes diarios, vehículo para movilización al sitio, libreta de campo, útiles de oficina.

\section{RESULTADOS}

\section{División político administrativa}

En el ámbito espacial la comunidad de Cunuyacu, se encuentra en la Parroquia de Pilahuín, Cantón Ambato de la provincia de Tungurahua, en la Región de Sierra de Ecuador (PDA Pilahuín, 2015, p. 1).

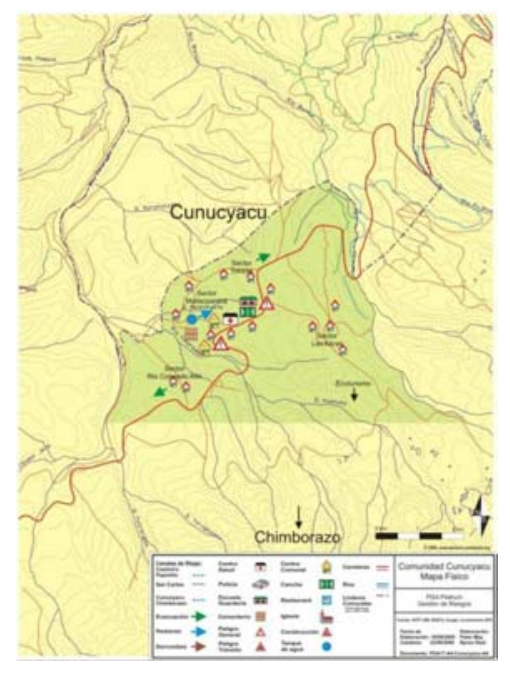

Figura 1. Mapa físico comunidad Cunuyacu.

Fuente: (PDA Pilahuín, 2015, p. 1).

\section{Población}

El 99\% de la población de Cunuyacu es indígena, siendo originarios de Kichwuas, el 1\% restante corresponde a población mestiza, algunos de ellos son hijos descendientes de los ascendados y mestizos provenientes de otras parroquias aledañas, en porcentaje y número de habitantes por sexo y etnia se presenta de la siguiente manera: socios comuneros 68 , mujeres 
ESTRATEGIA PARA MEJORAR LA CALIDAD DE VIDA DE LOS HABITANTES, CASO: COMUNIDAD CUNUYACU, PROVINCIA DE TUNGURAHUA (ECUADOR)

158, hombres 162, para un total de 320 habitantes, llevado a porcentaje tenemos $99 \%$ de indígenas y un $1 \%$ mestizos (COCAP, 2011, p. 23).

\section{Actividades económicas}

De la investigación de campo realizada, se pudo observar que la población se dedica principalmente al cultivo de productos agrícolas andinos como: papas, zanahoria, mellocos, ocas, ajo, cebada y al cultivo de pasto. La producción pecuaria se basa en la crianza de bovinos, ovinos y camélidos; además hay una importante producción de leche, lana y abono orgánico.

\section{Ocupación de los habitantes: (turismo)}

En el sector, se ofrecen tres locales de venta de comida rápida, las condiciones de los locales no son las adecuadas para recibir al turista, el complejo no tiene un mantenimiento adecuado debido a que no existe una persona encargado para realizar el trabajo, no existe una persona encargada de ventas de boletería, sino la modalidad de trabajo es por turno a cada uno de los socios, al complejo no se encuentran articulados ni se ofrecen ninguna actividad turística adicional a los baños termales.

\section{Condiciones ambientales de la zona}

El clima de área es de tipo ecuatorial de alta montaña, los suelos son negros, húmedos, presentan un relieve ondulado, cubierta con escasa vegetación (COCAP, 2011, p. 46).

\section{Análisis de la demanda}

En base a encuestas realizadas a turistas que visitan la Reserva de Producción de Fauna Chimborazo, la misma que articula al Complejo de Aguas Termales y son los posibles clientes. La muestra de estudio está conformada por 382 personas, de las cuales el 15\% lo constituyen los turistas extranjeros que provienen generalmente de Francia con un $16 \%$, el $12 \%$ son de España y Estados Unidos, respectivamente; con un 9\% cada uno Venezuela, Italia y Colombia como los más representativos. Mientras que el $85 \%$ son nacionales, estos provienen de todas las provincias del Ecuador, en mayor porcentaje de Chimborazo con el 30\%, Tungurahua con el $16 \%$, Pichincha con el $14 \%$ y Guayas con el $9 \%$, el porcentaje restante se distribuye entre las demás provincias. 
ESTRATEGIA PARA MEJORAR LA CALIDAD DE VIDA DE LOS HABITANTES, CASO: COMUNIDAD CUNUYACU, PROVINCIA DE TUNGURAHUA (ECUADOR)

El mercado nacional está compuesto en $51 \%$ del género femenino y en un $49 \%$ de individuos del género masculino; y en relación al mercado internacional el $46 \%$ del género femenino y el $54 \%$ lo conforman las personas del género masculino.

En relación al mercado nacional el $52 \%$ posee instrucción a un nivel superior, el $39 \%$ tiene instrucción de educación media, el $7 \%$ cuenta con educación básica y el $2 \%$ posee estudios de postgrados, mientras que en relación al mercado internacional el 60\% cuenta con estudios superiores, el $21 \%$ posee estudios de postgrados y el $19 \%$ cuenta con educación media.

Los feriados nacionales es la temporada en la que comúnmente viajan los turistas nacionales que representan un $40 \%$, el $37 \%$ lo realizan los fines de semana, el $16 \%$ en el período de vacaciones escolares de la sierra (julio-agosto) y el 6\% realizan los viajes entre semana. Mientras que los turistas internacionales prefieren viajar en un $44 \%$ los fines de semana, el $30 \%$ en periodo de vacaciones escolares (julio-agosto), el $14 \%$ entre semana y el $12 \%$ aprovechando los feriados.

La intención de visita de los turistas nacionales y extranjeros a la Reserva de Producción de Fauna Chimborazo y los servicios que requieren los turistas nacionales y extranjeros cuando viajan a un balneario (ver tabla 1 y 2 ).

\section{Tabla 1}

Intenciones de visita al sector

\begin{tabular}{ccccc}
\hline Detalle & \multicolumn{2}{c}{ Turistas Nacionales } & \multicolumn{2}{c}{ Turistas Extranjeros } \\
\cline { 2 - 5 } & Frecuencia absoluta & $\%$ & Frecuencia absoluta & $\%$ \\
\hline $\mathrm{Si}$ & 307 & 94 & 50 & 88 \\
$\mathrm{No}$ & 18 & 6 & 7 & 12 \\
\hline
\end{tabular}

Fuente: Elaboración propia. 
ESTRATEGIA PARA MEJORAR LA CALIDAD DE VIDA DE LOS HABITANTES, CASO: COMUNIDAD CUNUYACU, PROVINCIA DE TUNGURAHUA (ECUADOR)

Tabla 2

Servicios requeridos por los turistas

\begin{tabular}{lcccc}
\hline \multirow{2}{*}{ Detalle } & \multicolumn{2}{c}{ Turistas Nacionales } & \multicolumn{2}{c}{ Turistas Extranjeros } \\
\cline { 2 - 5 } & Frecuencia Absoluta & $\%$ & Frecuencia Absoluta & $\%$ \\
\hline $\begin{array}{l}\text { Servicio de } \\
\text { alimentación }\end{array}$ & 221 & 38 & 40 & 32 \\
$\begin{array}{l}\text { Servicio de } \\
\text { alojamiento }\end{array}$ & 51 & 9 & 20 & 16 \\
$\begin{array}{l}\text { Guianza } \\
\text { Transporte }\end{array}$ & 65 & 11 & 11 & 9 \\
$\begin{array}{l}\text { Sauna, turco, } \\
\text { hidromasaje }\end{array}$ & 54 & 9 & 28 & 22 \\
$\begin{array}{l}\text { Canchas } \\
\text { deportivas }\end{array}$ & 93 & 16 & 16 & 9 \\
\hline
\end{tabular}

Fuente: Elaboración propia.

\section{Análisis de la oferta y competencia}

La comunidad Cunuyacu, es poseedora de un atractivo propio de la zona y está articulada a dos grandes atractivos naturales de la provincia de Chimborazo (Reserva de Producción de Fauna Chimborazo y el Nevado Chimborazo).

Para realizar este análisis de la competencia, se consideró como competidores a todos aquellos lugares con características similares a las del Complejo Turístico de Aguas Termales Cunuyacu; se analizaron todos los lugares turísticos cercanos a la Comunidad los cuales ofrecen el servicio de Balnearios con piscinas de agua temperada, las aguas son frías y de vertientes naturales, más no existen fuentes de agua termal.

La investigación de mercado determinó que la competencia atrae a 26,400 clientes anuales, entre los que se pueden detallar: 9,504 adultos, 14,256 niños y niñas nacionales, 1,760 adultos y 880 niños y niñas extranjeras. 
ESTRATEGIA PARA MEJORAR LA CALIDAD DE VIDA DE LOS HABITANTES, CASO: COMUNIDAD

CUNUYACU, PROVINCIA DE TUNGURAHUA (ECUADOR)

\section{Confrontación Demanda Vs Oferta}

Realizado un balance entre la demanda y oferta al año 2016, se puede observar demanda insatisfecha de los servicios turísticos (ver tabla 3).

Tabla 3

Demanda insatisfecha

\begin{tabular}{ccc}
\hline \multicolumn{3}{c}{ Demanda Insatisfecha (DI) } \\
\hline 59209 & 11264 & DI \\
\hline Demanda potencial adultos & Competencia adultos & 47945 \\
Demanda potencial niños & Competencia niños & DI \\
80336 & 15136 & 65200 \\
\hline
\end{tabular}

Fuente: Elaboración propia.

Estudio mercadotécnico para el desarrollo del proyecto de turismo del balneario de aguas termales de la comunidad de Cunuyacu. Entre los aspectos estudiados con mayor importancia para el desarrollo del proyecto se tiene:

Viabilidad Técnica. El complejo turístico está diseñado para recibir a 50 turistas en las termas, 20 turistas en el área de piscina de entretenimiento y 30 personas en el área de sauna y turco. El proyecto funcionará al $70 \%$ de su demanda insatisfecha.

Viabilidad Económica y Financiera. El estudio económico y financiero estableció que el proyecto requerirá de una inversión inicial de \$255.310,95 de los cuales está propuesto realizar el financiamiento del 30\% con aporte de la Asociación; es decir, \$76.593,29 dólares; y el 70\% será financiado con la Corporación Financiera Nacional (CFN), lo que resulta \$178.717,67 dólares. Se determinaron los siguientes indicadores financieros en el escenario óptimo y con financiamiento (70\% demanda objetiva): Valor Actual Neto \$218.153,06; Tasa Interna de Retorno 41,13\% con respecto a la Tasa Mínima de Aceptable Rendimiento 13,49\%; Periodo de Recuperación 2,8 años respecto a lo proyectado cinco años; Relación Beneficio/Costo \$ 3,91. 
ESTRATEGIA PARA MEJORAR LA CALIDAD DE VIDA DE LOS HABITANTES, CASO: COMUNIDAD

CUNUYACU, PROVINCIA DE TUNGURAHUA (ECUADOR)

Viabilidad Ambiental. Los impactos negativos causados por la implementación de este proyecto son mínimos con respecto a los beneficios que contribuirá a la comunidad y al ambiente, los impactos no afectan de manera agresiva al ecosistema y con la implementación de buenas prácticas del turismo sostenible se puede contrarrestar los impactos ambientales causados, esto se puede lograr con la concientización y capacitación a la población y turistas en general.

\section{Verificación de la hipótesis}

La formulación de un proyecto de desarrollo turístico sostenible del Complejo de Aguas Termales de la comunidad Cunuyacu, mejorará la calidad de vida de sus habitantes.

H0: El proyecto de desarrollo turístico sostenible del Complejo de Aguas Termales de la comunidad Cunuyacu, NO mejora la calidad de vida de sus habitantes.

H1: El proyecto de desarrollo turístico sostenible del Complejo de Aguas Termales de la comunidad Cunuyacu, SI mejora la calidad de vida de sus habitantes.

$\mathrm{HO}=\mathrm{Hi}$

$\mathrm{HO} \neq \mathrm{Hi}$

Se trabajó con un nivel de significancia del $5 \%$

Prueba Chi Cuadrado X2

$$
\chi^{2}=\sum \frac{\left(o_{i}-e_{i}\right)^{2}}{e_{i}}
$$

En donde:

$\mathrm{O}=$ frecuencias observadas

e $=$ frecuencias esperadas

$\Sigma=$ sumatoria

La prueba de asociación o tabla de contingencia se encuentra en la siguiente tabla: 
ESTRATEGIA PARA MEJORAR LA CALIDAD DE VIDA DE LOS HABITANTES, CASO: COMUNIDAD CUNUYACU, PROVINCIA DE TUNGURAHUA (ECUADOR)

Tabla 4

Frecuencias observadas

\begin{tabular}{|c|c|c|c|c|c|}
\hline Encuesta a socios & $\mathrm{Si}$ & $\%$ & No & $\%$ & Total \\
\hline $\begin{array}{l}\text { ¿Considera usted que la formulación de } \\
\text { un proyecto de desarrollo turístico } \\
\text { sostenible para la implementación en el } \\
\text { Complejo de Aguas Termales de la } \\
\text { comunidad Cunuyacu mejora la calidad } \\
\text { de vida de sus habitantes? }\end{array}$ & 58 & 96,7 & 2 & 3,3 & 60 \\
\hline $\begin{array}{l}\text { ¿Está dispuesto a organizarse para } \\
\text { obtener mejores beneficios sociales y } \\
\text { económicos con el desarrollo turístico del } \\
\text { complejo? }\end{array}$ & 50 & 83,3 & 10 & 16,7 & 60 \\
\hline Total & 108 & 90 & 12 & 10 & 120 \\
\hline
\end{tabular}

Fuente: Elaboración propia.

\section{Cálculo de la frecuencia esperada}

$$
E 1=\frac{108 \times 60}{120}=54 \quad E 2=\frac{12 \times 60}{120}=6
$$


ESTRATEGIA PARA MEJORAR LA CALIDAD DE VIDA DE LOS HABITANTES, CASO: COMUNIDAD CUNUYACU, PROVINCIA DE TUNGURAHUA (ECUADOR)

Tabla 5

Frecuencias esperadas

\begin{tabular}{|c|c|c|c|c|c|}
\hline Encuesta a socios & $\mathrm{Si}$ & $\%$ & No & $\%$ & Total \\
\hline $\begin{array}{l}\text { ¿Considera usted que la formulación de } \\
\text { un proyecto de desarrollo turístico } \\
\text { sostenible para la implementación en el } \\
\text { Complejo de Aguas Termales de la } \\
\text { comunidad Cunuyacu mejora la calidad } \\
\text { de vida de sus habitantes? }\end{array}$ & 54 & 90 & 6 & 10 & 60 \\
\hline $\begin{array}{l}\text { ¿Está dispuesto a organizarse para } \\
\text { obtener mejores beneficios sociales y } \\
\text { económicos con el desarrollo turístico del } \\
\text { complejo? }\end{array}$ & 54 & 90 & 6 & 10 & 60 \\
\hline Total & 108 & 90 & 12 & 10 & 120 \\
\hline
\end{tabular}

Fuente: Elaboración propia.

\section{Cálculo Estadístico}

\section{Tabla 6}

Chi-cuadrado calculado $\left(X^{2} C\right)$

\begin{tabular}{|c|c|c|}
\hline \multicolumn{2}{|c|}{ Frecuencia } & Prueba \\
\hline $\mathrm{O}$ & $E$ & $(o-e) 2 / e$ \\
\hline 58 & 54 & 0,296 \\
\hline 2 & 6 & 2,660 \\
\hline 50 & 54 & 0,296 \\
\hline 10 & 6 & 2,660 \\
\hline Tot & $\left.{ }^{2} \mathrm{c}\right)$ & 5,910 \\
\hline
\end{tabular}

Fuente: Elaboración propia. 
ESTRATEGIA PARA MEJORAR LA CALIDAD DE VIDA DE LOS HABITANTES, CASO: COMUNIDAD

CUNUYACU, PROVINCIA DE TUNGURAHUA (ECUADOR)

\section{Deducción de conclusiones:}

Grados de libertad: Se obtuvo con la siguiente fórmula

$$
\mathrm{gl}=(f-1)(c-1)=(2-1)(2-1)=1
$$

Donde:

gl = grado de libertad

$f=$ filas

$\mathbf{c}=$ columnas

Grado de significación: Se utiliza un nivel de confianza de $95 \%$ y por el $5 \%$ de error para distribución unilateral, para distribución bilateral o de dos colas resulta 0,05/2=0,025.

Para obtener el Chi-cuadrado según la tabla de valores críticos, se buscó el grado de libertad y el nivel de confianza y así se obtuvo el Chi-cuadrado de la tabla (X2t) para una cola 3,84 y para dos colas 5,02 que se comparó con el Chi-cuadrado calculado $(X 2 c)=5,91$.

De acuerdo a este criterio se determinó las siguientes condiciones:

Xc $\geq X t$ Acepto la hipótesis de trabajo $(\mathrm{H} 1)$ y se rechaza la hipótesis nula $(\mathrm{H} 0)$

$\mathrm{Xc}<\mathrm{Xt}$ Se rechaza hipótesis de trabajo $(\mathrm{H} 1)$ y se acepta la hipótesis nula $(\mathrm{H} 0)$

Distribución Unilateral:

$X 2 c=5,91>X 2 t=3,84$

Distribución Bilateral:

$X 2 c=5,91>X 2 t=5,02$

De acuerdo a estos resultados se pudo comprobar que el Chi-cuadrado calculado es mayor que el Chi-cuadrado tabla, por lo cual se acepta la hipótesis de trabajo (H1) y se rechaza la hipótesis nula $(\mathrm{HO})$; es decir, "El proyecto de desarrollo turístico sostenible del Complejo de Aguas Termales de la comunidad Cunuyacu, SI mejora la calidad de vida de sus habitantes". 
ESTRATEGIA PARA MEJORAR LA CALIDAD DE VIDA DE LOS HABITANTES, CASO: COMUNIDAD CUNUYACU, PROVINCIA DE TUNGURAHUA (ECUADOR)

\section{DISCUSIÓN}

El diagnóstico de la situación actual de la comunidad Cunuyacu, permitió identificar los servicios básicos limitados en el sector rural, no hay cobertura para la interconectividad, infraestructura deteriorada, no acorde a la actividad turística, lo que dificulta un desarrollo más sostenible de los proyectos. El sector posee gran potencial de turismo comunitario y de crecimiento, dadas las fuentes atractivas naturales existentes, generando la posibilidad de ser un sitio turístico de gran afluencia de turistas nacionales y extranjeros. Se constituye un eje dinamizador de la economía local ayudando a mejorar las condiciones de vida de los habitantes de la zona, siendo factible la ejecución de proyectos de desarrollo turístico.

De acuerdo a Garrido, Villacís \& Cabanilla (2016), uno de los principales y quizás el mayor problema que tienen estos sitios de turismo comunitario, es la baja ocupación que tienen sus casas de alojamiento, sean estas exclusivas para turistas o las casas familiares de la comunidad. Se estima, por la información recibida que la tasa de ocupación no supera el 44\% en promedio, algo que el ámbito de alojamiento es preocupante. Las comunidades, especialmente las más pequeñas, pueden "cubrir esta brecha económica" con otros ingresos provenientes de sus actividades tradicionales: agricultura, ganadería y comercio de sus productos. Sin embargo, esto hace que naturalmente, su dedicación e inversión vayan con estas actividades, que les son más familiares y de las cuales han vivido siempre, descuidando el turismo como fuente de ingresos económicos. Este problema es el resultado de muchos errores cometidos en el proceso de desarrollo del turismo comunitario en el país. El primero, al parecer, fue la escasa o nula la planificación que se le dio a este tipo de turismo en sus inicios, ésta debió ser un modelo específico para desarrollar el turismo comunitario como destino turístico, y debió incluir metas a cumplir en plazos establecidos vinculando a todos los actores.

Los pobladores de la comunidad por dedicarse a las actividades tradicionales en agricultura y ganadería, no han explotado el turismo en la localidad. La actividad turística constituye un complemento a la economía de los habitantes de la población; la comunidad de Cunuyacu posee una variedad de recursos turísticos naturales, los mismos que pueden ser aprovechados de manera potencial para realizar turismo comunitario sustentable y sostenible en beneficio de toda la comunidad, mejorando sus ingresos económicos familiares y por derivación las condiciones de vida en salud, educación, generación de fuentes alternativas de ingresos, entre otros. 
ESTRATEGIA PARA MEJORAR LA CALIDAD DE VIDA DE LOS HABITANTES, CASO: COMUNIDAD CUNUYACU, PROVINCIA DE TUNGURAHUA (ECUADOR)

Para que el turismo de la localidad mejore en condiciones de oferta de servicios turísticos e infraestructura, es necesario que exista una alianza estratégica entre el sector público (Entidades estatales que lo promueven como el Ministerio de Turismo del Ecuador y las direcciones de turismo de los Gobiernos Autónomos Descentralizados de los cantones) y privado (La comunidad), como estrategia para mejorar las condiciones de vida de la gente. Para ello, es necesario diseñar planes, programas y proyectos debidamente estructurados y financiados, que sustenten la realización de nuevas inversiones.

\section{CONCLUSIONES}

Las principales actividades productivas son la agricultura y pecuarias. Existe un gran porcentaje de migración. No se realiza actividades asociadas al turismo, a más del balneario. Los habitantes no cuentan con sistemas de capacitación ni intercambio cultural en la comunidad. Están regidos por la Junta de Socios, dicha Asociación no posee un organigrama estructural y funcional organizado que les permita el desarrollo turístico.

De acuerdo a los resultados obtenidos, a los turistas tanto nacionales como extranjeros les gustaría realizar caminatas y observación de flora y fauna, por tanto se implementará recorridos por la comunidad visitando las actividades productivas que realizan los habitantes de la comunidad, como la observación de la práctica de ordeño manual de leche, que se puede apreciar a lo largo de la vía de ingreso al Campo Turístico.

Con la evaluación sociocultural y ambiental, se puede concluir que el proyecto producirá un impacto positivo a nivel cultural, social y ambiental.

\section{REFERENCIAS BIBLIOGRÁFICAS}

Baca, G. (2010). Evaluación de Proyectos. México D.F: McGraw-Hill.

Bernal, C. (2006). Metodología de la investigación: Para administración, economía, humanidades y ciencias sociales. México D.F: Pearson Educación.

Casas, F. (1999). Calidad de vida y calidad humana. Papeles del psicólogo, 74, pp. 46-54. 
ESTRATEGIA PARA MEJORAR LA CALIDAD DE VIDA DE LOS HABITANTES, CASO: COMUNIDAD

CUNUYACU, PROVINCIA DE TUNGURAHUA (ECUADOR)

COCAP. (2011). Plan de manejo de páramos de la zona alta de Pilahuín. Recuperado el 25 de agosto de 2015, de: http://rrnn.tungurahua.gob.ec/documentos/ver/520da475ebd424e01d000002.

Garrido, C., Villacis, C., \& Cabanilla, E. (2016). Turismo comunitario en el Ecuador: Un análisis sobre su crisis. Centro de Investigación y Desarrollo del Ecuador. Recuperado de: https://docs.wixstatic.com/ugd/54b18d_eb26adf172df4d0d90c7ac8116341d3f.pdf

Montes Castillo, M. D. L. L. (2006). Calidad de vida. Revista Mexicana de Medicina Física y Rehabilitación, 18(1), pp. 5-6.

PDA (PILAHUIN). (2011). Plan Comunitario de preparación para desastres. Recuperado el 4 de octubre de 2015. Recuperado de http://www.codeso.com/PDA-Pilahuin/Mapa-PDAPilahuin-Cunugyacu.html.

Plan Nacional del Buen Vivir. (2013, Julio). Recuperado de http://www.buenvivir.gob.ec/

Plandetur. (2007). Tourism \& Leisure. Recuperado de http://www.turismo.gob.ec/wpcontent/uploads/downloads/2013/02/PLANDETUR-2020.pdf

Estadísticas de llegada de extranjeros al Ecuador, (Enero, 2016). Revista Líderes. Ecuador. Recuperado de http://www.revistalìderes.ec/lìderes/pilares-sostienen-pib-ecuatorianos.html

Senplades. (2013) Plan Nacional Buen Vivir. Recuperado el 29 de abril de 2015 de http://www.buenvivir.gob.ec/.

Vara, A. (2012). Desde la idea inicial hasta la sustentación. 7 Pasos para una tesis exitosa. Un modelo efectivo para ciencias empresariales. Instituto de Investigación de la Facultad de Ciencias Administrativas y Recursos Humanos. Lima: Universidad de San Martín de Porres.

Varela, R. (2010) Evaluación económica de proyectos de inversión. Bogotá: McGraw-Hill.

World Tourism Organizations Network. Estados Unidos: Sustainable Development of Tourism. (2007). Recuperado el 10 de octubre de 2015, de http://sdt.unwto.org/es/content/definicion 